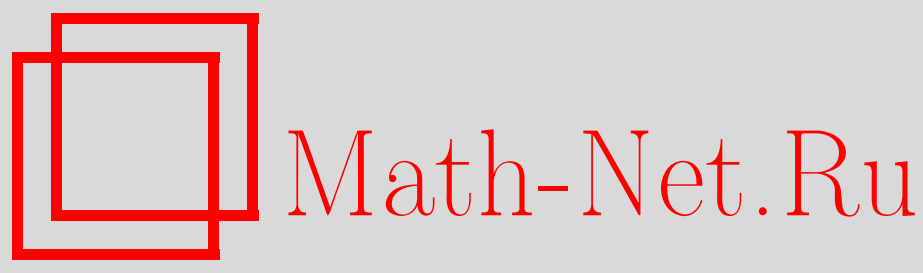

Ю. А. Церковников, Уравнения для двухвременных температурных функций Грина гейзенберговского ферромагнетика в приближении взаимодействующих мод, ТМФ, 2008, том 154, номер 1, 197-206

DOI: https://doi.org/10.4213/tmf6160

Использование Общероссийского математического портала Math-Net.Ru подразумевает, что вы прочитали и согласны с пользовательским соглашением http://www . mathnet.ru/rus/agreement

Параметры загрузки:

IP : 54.237 .59 .107

26 апреля 2023 г., 10:33:21

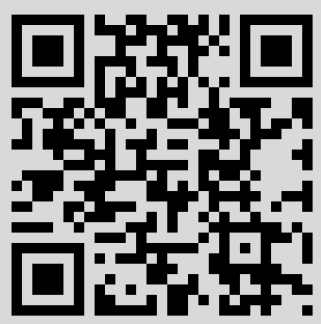




\section{УРАВНЕНИЯ ДЛЯ ДВУХВРЕМЕННЫХ ТЕМПЕРАТУРНЫХ ФУНКЦИЙ ГРИНА ГЕЙЗЕНБЕРГОВСКОГО ФЕРРОМАГНЕТИКА В ПРИБЛИЖЕНИИ ВЗАИМОДЕЙСТВУЮЩИХ МОД}

В приближении взаимодействующих мод получена замкнутая система уравнений для функций Грина поперечных и продольных компонент спина, точно учитывающая правила сумм, связанных с конечностью спина.

Ключевые слова: приближение взаимодействующих мод, правила сумм для спиновых операторов.

К 90-летию со дня рождения Д. Н. Зубарева

\section{1. ОСНОВНЫЕ СООТНОШЕНИЯ}

В работе рассмотрен анизотропный (типа “легкая ось") гейзенберговский ферромагнетик со спином $1 / 2$, гамильтониан которого в импульсном представлении имеет вид

$$
\mathcal{H}=-\mu H \sqrt{N} s_{0}^{z}-\frac{1}{2} \sum_{k} I(k)\left\{\xi s_{k}^{-} s_{k}^{+}+s_{k}^{z} s_{-k}^{z}\right\},
$$

где $H$ - внешнее магнитное поле; $\mu=g \mu_{\mathrm{B}}, g \approx 2 ; \mu_{\mathrm{B}}$ - магнетон Бора; $\xi$ - параметр анизотропии, $\xi \leqslant 1 ; I(k)=I(0) \gamma_{k}, I(0)>0 ; \gamma_{k}=z^{-1} \sum_{\delta} e^{i k \delta} ; z-$ число ближайших соседей в узле кристаллической решетки; $\delta$ - ненулевые векторы, определяющие их положение относительно выбранного узла $f$. Мы ограничиваемся случаем простой кубической решетки с постоянной $a$.

Фурье-образы спиновых операторов $s_{k}^{ \pm}=s_{k}^{x} \pm s_{k}^{y}, s_{k}^{z}$ в узлах $f$ задаются формулами

$$
s_{k}^{ \pm}=\frac{1}{\sqrt{N}} \sum_{f} s_{f}^{+} e^{ \pm i f k}, \quad s_{k}^{z}=\frac{1}{\sqrt{N}} \sum_{f} s_{f}^{z} e^{i f k}
$$

где $N$ - число узлов решетки.

Перестановочные соотношения для операторов (1.2) имеют вид

$$
\left[s_{k}^{+}, s_{k^{\prime}}^{-}\right]_{-}=\frac{2}{\sqrt{N}} s_{k-k^{\prime}}^{z}, \quad\left[s_{k}^{ \pm}, s_{k^{\prime}}^{z}\right]_{-}=\mp \frac{1}{\sqrt{N}} s_{k \pm k^{\prime}}^{ \pm}, \quad\left[s_{k}^{ \pm}, s_{k^{\prime}}^{ \pm}\right]_{-}=0 .
$$

* Математический институт им. В. А. Стеклова РАН, Москва, Россия 
Кроме того, вследствие конечности состояний спина в узле $f$ операторы $s_{k}^{+}, s_{k}^{-}$, и $s_{k}^{z}$ подчиняются более сложным, чем бозе- и ферми-операторы, кинематическим соотношениям. В случае спина $s=1 / 2$ эти соотношения имеют вид

$$
\begin{aligned}
s_{k}^{+} & =\frac{2}{\sqrt{N}} \sum_{k^{\prime}} s_{k^{\prime}}^{z} s_{-k^{\prime}+k}^{+}=-\frac{2}{\sqrt{N}} \sum_{k^{\prime}} s_{-k^{\prime}+k}^{+} s_{k^{\prime}}^{z}, \\
s_{-k}^{-} & =\frac{2}{\sqrt{N}} \sum_{k^{\prime}} s_{k^{\prime}-k}^{-} s_{k^{\prime}}^{z}=-\frac{2}{\sqrt{N}} \sum_{k^{\prime}} s_{k^{\prime}}^{z} s_{k^{\prime}-k}^{-}, \\
s_{k}^{z} & =\frac{\sqrt{N}}{2} \delta_{k, 0}-\frac{1}{\sqrt{N}} \sum_{k^{\prime}} s_{k^{\prime}}^{-} s_{k^{\prime}+k}^{+}=-\frac{\sqrt{N}}{2} \delta_{k, 0}+\frac{1}{\sqrt{N}} \sum_{k^{\prime}} s_{k^{\prime}+k}^{+} s_{k^{\prime}}^{-}= \\
& =\frac{1}{2 \sqrt{N}} \sum_{k^{\prime}}\left(s_{k^{\prime}+k}^{+} s_{k^{\prime}}^{-}-s_{k^{\prime}}^{-} s_{k^{\prime}+k}^{+}\right), \\
\frac{1}{\sqrt{N}} \sum_{k^{\prime}} s_{k^{\prime}}^{z} s_{-k^{\prime}+k}^{z} & =\frac{1}{4} \delta_{k, 0}, \quad \frac{1}{\sqrt{N}} \sum_{k^{\prime}} s_{k^{\prime}}^{-} s_{-k^{\prime}+k}^{-}=\frac{1}{\sqrt{N}} \sum_{k^{\prime}} s_{k^{\prime}}^{+} s_{-k^{\prime}+k}^{+}=0 .
\end{aligned}
$$

В (1.4) суммирования по $k^{\prime}$ производятся по первой приведенной зоне Бриллюена.

Дифференцируя по времени операторы $s_{q}^{+}(t)$ и $s_{q}^{z}(t)$, записанные в гейзенберговском представлении $\left(s(t)=e^{i t \mathcal{H}} \cdot s \cdot e^{-i t \mathcal{H}}\right)$, получаем бесконечную цепочку зацепляющихся уравнений. Для $s_{q}^{+}(t)$ имеем

$$
\begin{gathered}
i \frac{d}{d t} s_{q}^{+}=\left[s_{q}^{+}, \mathcal{H}\right]_{-}=\mu H s_{q}^{+}+\frac{I(0)}{\sqrt{N}} \sum_{k}\left(\gamma_{k}-\xi \gamma_{-k+q}\right) s_{k}^{z} s_{-k+q}^{+}, \\
i \frac{d}{d t} s_{k}^{z} s_{-k+q}^{+}=\left[s_{k}^{z} s_{-k+q}^{+}, \mathcal{H}\right]_{-}=\mu H s_{k}^{z} s_{-k+q}^{+}+ \\
+\frac{I(0)}{\sqrt{N}} \sum_{k}\left\{\left(\gamma_{k}-\xi \gamma_{-k_{1}-k+q}\right) s_{k}^{z} s_{k_{1}}^{z} s_{-k_{1}-k+q}^{+}\right. \\
\left.-\frac{\xi}{2}\left(\gamma_{k_{1}}-\gamma_{k_{1}+k}\right) s_{k_{1}}^{-} s_{k_{1}+k}^{+} s_{-k+q}^{+}\right\}
\end{gathered}
$$

и т.д.; для $s_{q}^{z}(t)$ имеем

$$
\begin{gathered}
i \frac{d}{d t} s_{k}^{z}=\left[s_{k}^{z}, \mathcal{H}\right]_{-}=-\frac{\xi I(0)}{2 \sqrt{N}} \sum_{k}\left(\gamma_{k}-\gamma_{-k+q}\right) s_{-k}^{-} s_{-k+q}^{+} \\
i \frac{d}{d t} s_{-k}^{-} s_{-k+q}^{+}=\left[s_{-k}^{-} s_{-k+q}^{+}, \mathcal{H}\right]_{-}=\frac{I(0)}{\sqrt{N}} \sum_{k}\left\{\left(\gamma_{k_{1}}-\xi \gamma_{-k_{1}-k+q}\right) s_{-k}^{-} s_{k_{1}}^{z} s_{-k_{1}-k+q}^{+}\right. \\
\left.\quad-\left(\gamma_{k_{1}}-\xi \gamma_{-k_{1}+k}\right) s_{-k+k_{1}}^{-} s_{k_{1}+k}^{z} s_{-k+q}^{+}\right\}
\end{gathered}
$$

и т.д. (для простоты записи зависимость операторных уравнений от $t$ в (1.5)-(1.8) не указана). 
При построении уравнений для двухвременных температурных функций Боголюбова-Тябликова-Зубарева (см. работы [1]-[3]) воспользуемся методом дифференцирования по двум временам [4] (см. также раздел 2 работы [5]). Будем рассматривать функции Грина двух видов: запаздывающие коммутаторные функции

$$
\left\langle\left\langle A \mid B^{+}\right\rangle\right\rangle_{E}=-i \int_{0}^{\infty} d t e^{i E t}\left\langle\left[A(t), B^{+}\right]_{-}\right\rangle, \quad \operatorname{Im} E>0,
$$

и Фурье-образы функции релаксации Кубо [6]

$$
\left(\left(A \mid B^{+}\right)\right)_{E}=-i \int_{0}^{\infty} d t e^{i E t}\left(A(t) \mid B^{+}\right), \quad \operatorname{Im} E>0
$$

где

$$
\left(A(t) \mid B^{+}\right)=i \int_{t}^{\infty} d t^{\prime}\left\langle\left[A\left(t^{\prime}\right), B^{+}\right]_{-}\right\rangle
$$

- функция, обладающая свойствами скалярного произведения. В дальнейшем мы полагаем, что выполнены условия эргодичности [6], и функция $\left(A \mid B^{+}\right)$также равна $(A \mid B)^{T}=\int_{0}^{\beta} d \tau\left\langle\Delta A \cdot \Delta B^{+}(\tau)\right\rangle$, где $\beta=1 /(k T), \Delta A=A-\langle A\rangle, \Delta B^{+}=B^{+}-\left\langle B^{+}\right\rangle$.

Согласно методу работы [4] для последовательности операторов (или столбцов операторов) $A_{1}, A_{2}, \ldots$ получается бесконечная система уравнений (по существу, тождеств)

$$
\begin{aligned}
E\left\langle\left\langle A_{n} \mid A_{n}^{+}\right\rangle\right\rangle_{E, n-1}=\langle & A_{n}\left|A_{n}\right\rangle+\left\{\left\langle i \dot{A}_{n} \mid A_{n}^{+}\right\rangle+\left\langle\left\langle i \dot{A}_{n} \mid-i \dot{A}_{n}^{+}\right\rangle\right\rangle_{E, n}\right\} \times \\
& \times\left\langle A_{n} \mid A_{n}^{+}\right\rangle^{-1}\left\langle\left\langle A_{n} \mid A_{n}^{+}\right\rangle\right\rangle_{E, n-1}, \quad n=1,2, \ldots,
\end{aligned}
$$

$A_{1}$ - исходный оператор (или столбец операторов), определяющий поставленную задачу, и $A_{2}, A_{3}, \ldots$ - операторы входящие соответственно в производные $i \dot{A}_{1}=$ $\left[A_{1}, \mathcal{H}\right]_{-}, i \dot{A}_{2}=\left[A_{2}, \mathcal{H}\right]_{-}, \ldots$. Операторы $A_{2}, A_{3}, \ldots$ последовательно ортогонализованы относительно скалярных произведений $\left\langle A_{1} \mid A_{1}^{+}\right\rangle,\left\langle A_{2} \mid A_{2}^{+}\right\rangle, \ldots$, поэтому $\left\langle A_{n} \mid A_{n}^{+}\right\rangle_{n-1}=\left\langle A_{n} \mid A_{n}\right\rangle$. В (1.12) коммутаторные функции, отмеченные индексом $n$, определяются рекуррентными соотношениями

$$
\left\langle\left\langle A \mid B^{+}\right\rangle\right\rangle_{E, n}=\left\langle\left\langle A \mid B^{+}\right\rangle\right\rangle_{E, n-1}-\left\langle\left\langle A \mid A_{n}^{+}\right\rangle\right\rangle_{E, n-1}\left\langle\left\langle A_{n} \mid A_{n}^{+}\right\rangle\right\rangle_{E, n-1}^{-1}\left\langle\left\langle A_{n} \mid B^{+}\right\rangle\right\rangle_{E, n-1}
$$

и обладают свойством неприводимости, поскольку $\left\langle\left\langle A_{m} \mid B^{+}\right\rangle\right\rangle_{E, n}=\left\langle\left\langle A \mid A_{m}^{+}\right\rangle\right\rangle_{E, n}=0$ при $m \leqslant n$. Функции (1.9) и (1.10) связаны простым соотношением

$$
\left(\left(A \mid B^{+}\right)\right)_{E}=\frac{\left\langle\left\langle A \mid B^{+}\right\rangle\right\rangle_{E}+\left(A \mid B^{+}\right)}{E},
$$

где $\chi_{A B^{+}}=\left(A \mid B^{+}\right)=\left\langle\left\langle A \mid B^{+}\right\rangle\right\rangle_{E=0}-$ величина, имеющая смысл восприимчивости.

\section{2. УРАВНЕНИЯ ДЛЯ ОДНОЧАСТИЧНЫХ ФУНКЦИЙ ГРИНА}

Исходя из уравнений движения (1.5) и (1.7) мы можем получить цепочки уравнений как для коммутаторных функций (1.9), так и для функций релаксации (1.10). Функции релаксации (1.10) предпочтительнее, поскольку для них уравнения более симметричны относительно $s_{k}^{ \pm}$и $s_{k}^{z}$ в силу того, что начальные условия (при $\left.t=0\right)$ 
для $\left(s_{k}^{+} \mid s_{k}^{-}\right)$и $\left(s_{k}^{z} \mid s_{-k}^{z}\right)$ отличны от нуля. Поэтому функции релаксации более удобны для рассмотрения как вблизи температуры $\theta=k T \cong 0$, так и при $\theta \neq 0$. Для коммутаторных функции (1.9) имеем $\left\langle\left[s_{k}^{+} s_{k}^{-}\right]_{-}\right\rangle=2\left\langle s_{k=0}^{z}\right\rangle / \sqrt{N}$, тогда как $\left\langle\left[s_{k}^{z}, s_{-k}^{z}\right]_{-}\right\rangle=0$, и эта симметрия теряется. В дальнейшем мы будем в основном рассматривать получающиеся из (1.12) первые уравнения цепочек

$$
\begin{aligned}
\left(\left(s_{q}^{+} \mid s_{q}^{-}\right)\right)_{E} & =\frac{\left(s_{q}^{+} \mid s_{q}^{-}\right)}{E-\left(i \dot{s}_{q}^{+} \mid s_{q}^{-}\right) /\left(s_{q}^{+} \mid s_{q}^{-}\right)-\left(\left(i \dot{s}_{q}^{+} \mid-i \dot{s}_{q}^{-}\right)\right)_{1, E} /\left(s_{q}^{+} \mid s_{q}^{-}\right)}, \\
\left(\left(s_{q}^{z} \mid s_{-q}^{z}\right)\right)_{E} & =\frac{\left(s_{q}^{z} \mid s_{-q}^{z}\right)}{E-\left(\left(i \dot{s}_{q}^{z} \mid-i \dot{s}_{-q}^{z}\right)\right)_{1, E} /\left(s_{q}^{z} \mid s_{-q}^{z}\right)},
\end{aligned}
$$

где $\left(i \dot{s}_{q}^{+} \mid s_{q}^{-}\right)=\left\langle\left[s_{q}^{+}, s_{q}^{-}\right]_{-}\right\rangle=2 \sigma, \sigma=\left\langle s_{0}^{z}\right\rangle / \sqrt{N}$ - намагниченность в узле $f$ (спонтанная при $H=0),\left(i \dot{s}_{q}^{z} \mid s_{-q}^{z}\right)=\left\langle\left[s_{q}^{z}, s_{-q}^{z}\right]_{-}\right\rangle=0$. В (2.1) и (2.2) неприводимые функции ("массовые операторы") равны

$$
\begin{aligned}
\left(\left(i \dot{s}_{q}^{+} \mid i \dot{s}_{q}^{-}\right)\right)_{1, E}= & \frac{I^{2}(0)}{N} \sum_{k, k^{\prime}}\left(\gamma_{k}-\xi \gamma_{-k+q}\right)\left(\gamma_{k^{\prime}}-\xi \gamma_{-k^{\prime}+q}\right) \times \\
& \times\left(\left(s_{k}^{z} s_{-k+q}^{+} \mid s_{-k^{\prime}+q^{-}}^{-} s_{-k^{\prime}}^{z}\right)\right)_{1, E}, \\
\left(\left(i \dot{s}_{q}^{z} \mid-i \dot{s}_{-q}^{z}\right)\right)_{1, E}= & \frac{\xi^{2} I^{2}(0)}{4 N} \sum_{k, k^{\prime}}\left(\gamma_{k}-\gamma_{-k+q}\right)\left(\gamma_{k^{\prime}}-\gamma_{-k^{\prime}+q}\right) \times \\
& \times\left(\left(s_{-k}^{-} s_{-k+q}^{+} \mid s_{-k^{\prime}+q}^{-} s_{k^{\prime}}^{+}\right)\right)_{1, E}
\end{aligned}
$$

и не содержат согласно (1.13) частей, сводящихся соответственно к $s_{k}^{+}, s_{k}^{-}$и $s_{k}^{z}$. Уравнения (2.1) и (2.2) удобно переписать, введя в левые части с помощью (1.14) вместо функций релаксации коммутаторные функции

$$
\begin{aligned}
\left\langle\left\langle s_{q}^{+} \mid s_{q}^{-}\right\rangle\right\rangle_{E} & =\frac{2 \sigma+\left(\left(i \dot{s}_{q}^{+} \mid-i \dot{s}_{q}^{-}\right)\right)_{1, E}}{E-\left(2 \sigma+\left(\left(i \dot{s}_{q}^{+} \mid-i \dot{s}_{q}^{-}\right)\right)_{1, E}\right) /\left(s_{q}^{+} \mid s_{q}^{-}\right)}, \\
\left\langle\left\langle s_{q}^{z} \mid s_{-q}^{z}\right\rangle\right\rangle_{E} & =\frac{\left(\left(i \dot{s}_{q}^{z} \mid-i \dot{s}_{-q}^{z}\right)\right)_{1, E}}{E-\left(\left(i \dot{s}_{q}^{z} \mid-i \dot{s}_{-q}^{z}\right)\right)_{1, E} /\left(s_{q}^{z} \mid s_{-q}^{z}\right)} .
\end{aligned}
$$

В равенствах (2.5) и (2.6) можно произвести сдвиг операторов $s_{k}^{z}$ на величину намагниченности: $s_{k}^{z}=\delta_{k, 0} \sqrt{N} \sigma+\delta s_{k}^{z}\left(\left\langle\delta s_{k}^{z}\right\rangle=0\right)$. При этом $i \dot{s}_{q}^{z}=i \delta \dot{s}_{q}^{z}$ и $\left(s_{q}^{z} \mid s_{-q}^{z}\right)=$ $\left(\delta s_{q}^{z} \mid \delta s_{-q}^{z}\right)($ см. равенство (1.11))

Уравнения для восприимчивостей. В уравнения (2.1)-(2.6) вошли не определенные пока статические восприимчивости $\left(s_{q}^{+} \mid s_{q}^{-}\right)$и $\left(s_{q}^{z} \mid s_{-q}^{z}\right)$. Покажем, что мы можем их получить из коммутаторных функций Грина (1.9), взятых при $E=$ 0 , если воспользуемся тем, что скалярные произведения операторов $A$ и $B^{+}$равны $\left(A \mid B^{+}\right)=-\left\langle\left\langle A \mid B^{+}\right\rangle\right\rangle_{E=0}$. За основу возьмем функции Грина $\left\langle\left\langle\delta s_{k}^{z} s_{-k+q}^{+} \mid s_{q}^{-}\right\rangle\right\rangle_{E}$ и $\left\langle\left\langle s_{-k}^{-} s_{-k+q}^{+} \mid \delta s_{-q}^{z}\right\rangle\right\rangle_{E}$, построенные на уравнениях движения второго звена цепочек (1.6) и (1.8). Для них начальные условия (коммутаторы $\left\langle\left[\delta s_{k}^{z} s_{-k+q}^{+}, s_{q}^{-}\right]_{-}\right\rangle$и $\left.\left\langle\left[s_{-k}^{-} s_{-k+q}^{+}, \delta s_{-q}^{z}\right]_{-}\right\rangle\right)$отличны от нуля при всех значениях температуры $\theta=k T$. Напротив, для функции $\left\langle\left\langle s_{q}^{+} \mid s_{q}^{-}\right\rangle\right\rangle$начальное условие $\left\langle\left[s_{q}^{+}, s_{q}^{-}\right]_{-}\right\rangle=2 \sigma$ является ненулевым только при $\theta<\theta_{\text {кр }}$, а для $\left\langle\left\langle s_{q}^{z} \mid s_{-q}^{z}\right\rangle\right\rangle$ всегда равно нулю $\left(\left\langle\left[s_{q}^{z}, s_{-q}^{z}\right]_{-}\right\rangle=0\right)$. 
Умножая уравнения для $\left\langle\left\langle\delta s_{k}^{z} s_{-k+q}^{+} \mid s_{q}^{-}\right\rangle\right\rangle_{E}$ и $\left\langle\left\langle s_{-k}^{-} s_{-k+q}^{+} \mid \delta s_{-q}^{z}\right\rangle\right\rangle_{E}$ соответственно на $I(0)\left(\gamma_{k}-\xi \gamma_{-k+q}\right) / \sqrt{N}$ и $I(0)\left(\gamma_{k}-\gamma_{-k+q}\right) / \sqrt{N}$ и суммируя по $k$ при $E=0$, получаем (см. (1.6) и (1.8))

$$
\begin{aligned}
& \frac{I(0)}{\sqrt{N}} \sum_{k}\left(\gamma_{k}-\xi \gamma_{-k+q}\right)\left\langle\left[s_{k}^{z} s_{-k+q}^{+}, s_{q}^{-}\right]_{-}\right\rangle= \\
& =\frac{I(0)}{\sqrt{N}} \sum_{k}\left(\gamma_{k}-\xi \gamma_{-k+q}\right) \omega_{-k+q}^{T}\left(\delta s_{k}^{z} s_{-k+q}^{+} \mid s_{q}^{-}\right)+ \\
& +\frac{I^{2}(0)}{N} \sum_{k, k_{1}}\left(\gamma_{k}-\xi \gamma_{-k+q}\right)\left\{\left(\gamma_{k_{1}}-\xi \gamma_{-k_{1}-k+q}\right)\left(\delta s_{k}^{z} \delta s_{k_{1}}^{z} s_{-k_{1}-k+q}^{+} \mid s_{q}^{-}\right)-\right. \\
& \left.-\frac{\xi}{2}\left(\gamma_{k_{1}}-\gamma_{-k_{1}+k}\right)\left(s_{-k_{1}}^{-} s_{-k_{1}+k}^{+} s_{-k+q}^{+} \mid s_{q}^{-}\right)\right\} \\
& \frac{I(0)}{\sqrt{N}} \sum_{k}\left(\gamma_{k}-\gamma_{-k+q}\right)\left\langle\left[s_{-k}^{-} s_{-k+q}^{+}, s_{-q}^{z}\right]_{-}\right\rangle= \\
& =\sigma \frac{\xi I^{2}(0)}{\sqrt{N}} \sum_{k}\left(\gamma_{k}-\gamma_{-k+q}\right)^{2}\left(s_{-k}^{-} s_{-k+q}^{+} \mid \delta s_{-q}^{z}\right)+ \\
& +\frac{I^{2}(0)}{N} \sum_{k, k_{1}}\left\{\left(\gamma_{k}-\gamma_{-k+q}\right)\left(\gamma_{k_{1}}-\xi \gamma_{-k_{1}-k+q}\right)-\right. \\
& \left.-\left(\gamma_{k+k_{1}}-\gamma_{-k_{1}-k+q}\right)\left(\gamma_{k_{1}}-\xi \gamma_{k}\right)\right\}\left(s_{-k}^{-} \delta s_{k_{1}}^{z} s_{-k_{1}-k+q}^{+} \mid \delta s_{-q}^{z}\right),
\end{aligned}
$$

где $\omega_{q}^{T}=\mu H+\sigma I(0)\left(1-\xi \gamma_{q}\right)-$ перенормированная энергия магнона [1].

Повысив с помощью (1.4) порядок спиновых произведений в первых членах правых частей (2.7) и (2.8), получим

$$
\begin{aligned}
& \frac{I(0)}{\sqrt{N}} \sum_{k}\left(\gamma_{k}-\xi \gamma_{-k+q}\right) \omega_{-k+q}^{T}\left(\delta s_{k}^{z} s_{-k+q}^{+} \mid s_{q}^{-}\right)= \\
& \quad=\frac{2 I(0)}{N} \sum_{k, k_{1}}\left(\gamma_{k}-\xi \gamma_{-k+q}\right) \omega_{-k+q}^{T}\left(\delta s_{k}^{z} s_{k_{1}}^{z} s_{-k_{1}-k+q}^{+} \mid s_{q}^{-}\right)= \\
& \quad=\frac{2}{1-2 \sigma} \frac{I(0)}{N} \sum_{k, k_{1}}\left(\gamma_{k}-\xi \gamma_{-k+q}\right) \omega_{-k+q}^{T}\left(\delta s_{k}^{z} \delta s_{k_{1}}^{z} s_{-k_{1}-k+q}^{+} \mid s_{q}^{-}\right), \\
& \sigma \frac{\xi I^{2}(0)}{\sqrt{N}} \sum_{k}\left(\gamma_{k}-\gamma_{-k+q}\right)^{2}\left(s_{-k}^{-} s_{-k}^{+} \mid \delta s_{-q}^{z}\right)= \\
& \quad=2 \sigma \frac{\xi I^{2}(0)}{N} \sum_{k, k_{1}}\left(\gamma_{k}-\gamma_{-k+q}\right)^{2}\left(s_{-k}^{-} s_{k_{1}}^{z} s_{-k_{1}-k+q}^{+} \mid \delta s_{-q}^{z}\right)= \\
& \quad=\frac{2 \sigma}{1-2 \sigma} \frac{\xi I^{2}(0)}{N} \sum_{k, k_{1}}\left(\gamma_{k}-\gamma_{-k+q}\right)^{2}\left(s_{-k}^{-} \delta s_{k_{1}}^{z} s_{-k_{1}-k+q}^{+} \mid \delta s_{-q}^{z}\right)
\end{aligned}
$$

Произведем теперь спаривания спиновых операторов $\left\langle s_{k}^{-} s_{k}^{+}\right\rangle$и $\left\langle\delta s_{k}^{z} \delta s_{-k}^{z}\right\rangle$ в правых частях $(2.7),(2.8)$ (с учетом $(2.9),(2.10))$ и выделим тем самым скалярные произведе- 
ния $\left(s_{q}^{+} \mid s_{q}^{-}\right)$и $\left(\delta s_{q}^{z} \mid \delta s_{-q}^{z}\right)$; в результате получим связь восприимчивостей с усредненными коммутаторами в левых частях $(2.7),(2.8)$. Однако прежде чем производить спаривания в (2.7)-(2.10), необходимо учесть снижение порядка спиновых произведений в совпадающих узлах, обусловленное конечностью состояний спина. В импульсном представлении произведениям в совпадающих узлах соответствуют свертки произведений операторов (1.4) (см. также работу [8]). Только после этого можно производить спаривания операторов в правых частях (2.7)-(2.10). В оставшихся при этом произведениях, отмеченных точками $\left(: \delta s_{k}^{z} \delta s_{k_{1}}^{z} s_{-k_{1}-k+q}^{+}:, s_{-k_{1}}^{-} s_{-k_{1}+k}^{+} s_{-k+q}^{+}\right.$: и $\left.: s_{-k}^{-} \delta s_{k_{1}}^{z} s_{-k_{1}-k+q}^{+}:\right)$, спаривания запрещены. В приближении взаимодействующих мод мы полагаем

$$
\begin{gathered}
\left(: \delta s_{k}^{z} \delta s_{k_{1}}^{z} s_{-k_{1}-k+q}^{+}: \mid s_{q}^{-}\right) \cong 0, \quad\left(: s_{-k_{1}}^{-} s_{-k_{1}-k}^{+} s_{-k+q}^{+}: \mid s_{q}^{-}\right) \cong 0 \\
\left(: s_{-k}^{-} \delta s_{k_{1}}^{z} s_{-k_{1}-k+q}^{+}: \mid \delta s_{-q}^{z}\right) \cong 0 .
\end{gathered}
$$

Опустим довольно громоздкие выкладки и приведем окончательные (с учетом (2.11)) результаты для поперечной $\left(s_{q}^{+} \mid s_{q}^{-}\right)$и продольной $\left(\delta s_{q}^{z} \mid \delta s_{-q}^{z}\right)$ восприимчивостей (ср. с формулами (49)-(53) работы [8] при $\theta>\theta_{\text {кр }}$ ):

$$
\begin{aligned}
\left(s_{q}^{+} \mid s_{q}^{-}\right)^{-1} I(0)\left\{\left(1-\xi \gamma_{q}\right) \nu^{(1)}+\left(\xi-\gamma_{q}\right) n^{(1)}\right\}=\frac{I^{2}(0)}{2}\left(1-\xi \gamma_{q}\right) \times \\
\quad \times\left\{\frac{I^{-1}(0)}{N} \sum_{k} \omega_{-k+q}^{T} \gamma_{k}\left\langle\delta s_{k}^{z} \delta s_{-k}^{z}\right\rangle-\right. \\
\left.\quad-\sigma \frac{\xi}{z} n^{(1)}+\left(1-\xi \gamma_{q}\right) \nu^{(1)}+\nu^{(2)}-\nu^{(1)}-\frac{\xi}{z} n^{(1)}+\frac{2}{z} n(1-n)\right\}+ \\
\quad+\frac{I^{2}(0)}{2}\left(\xi-\gamma_{q}\right)\left\{\frac{I^{-1}(0)}{N} \sum_{k} \omega_{k}^{T} \gamma_{k}\left\langle s_{-k}^{-} s_{-k}^{+}\right\rangle-\right. \\
\left.\quad-\sigma \frac{\xi}{2 n z} \nu^{(1)}+\left(1-\xi \gamma_{q}\right) n^{(1)}+\xi n^{(2)}-n^{(1)}-\frac{\xi}{z} \nu^{(1)}+\frac{\xi}{z} n\right\} \\
\left(s_{q}^{z} \mid s_{-q}^{z}\right)^{-1} I(0)\left(1-\gamma_{q}\right) n^{(1)}=\xi I^{2}(0)\left(1-\gamma_{q}\right)\left\{\frac{\xi}{2}\left(1-\gamma_{q}\right) \frac{1}{N} \sum_{k} \gamma_{k}^{2}\left\langle s_{-k}^{-} s_{-k}^{+}\right\rangle+\right. \\
\left.\quad+\left(1-\gamma_{q}\right) n^{(1)}+\xi n^{(2)}-\left(1+\frac{1}{z}\right) n^{(1)}+\frac{\xi}{z} n\right\} .
\end{aligned}
$$

В (2.12) и (2.13) введены следующие обозначения:

$$
\begin{gathered}
\sigma=\frac{1}{2}-n, \quad n=\frac{1}{N} \sum_{k}\left\langle s_{k}^{-} s_{k}^{+}\right\rangle, \quad n^{(1)}=\frac{1}{N} \sum_{k} \gamma_{k}\left\langle s_{k}^{-} s_{k}^{+}\right\rangle, \\
n^{(2)}=\frac{1}{N} \sum_{k} \gamma_{k}^{2}\left(\left\langle s_{k}^{-} s_{k}^{+}\right\rangle-n\right)=\frac{1}{N} \sum_{k}\left(\gamma_{k}^{2}-\frac{1}{z}\right)\left\langle s_{k}^{-} s_{k}^{+}\right\rangle, \\
\nu=\frac{2}{N} \sum_{k}\left\langle\delta s_{k}^{z} \delta s_{-k}^{z}\right\rangle=2 n(1-n)=\frac{1-4 \sigma^{2}}{2}, \quad \nu^{(1)}=\frac{2}{N} \sum_{k} \gamma_{k}\left\langle\delta s_{k}^{z} \delta s_{-k}^{z}\right\rangle, \\
\nu^{(2)}=\frac{1}{N} \sum_{k} \gamma_{k}^{2}\left(2\left\langle\delta s_{k}^{z} \delta s_{-k}^{z}\right\rangle-n(1-n)\right)=\frac{2}{N} \sum_{k}\left(\gamma_{k}^{2}-\frac{1}{z}\right)\left\langle\delta s_{k}^{z} \delta s_{-k}^{z}\right\rangle,
\end{gathered}
$$


где $N^{-1} \sum_{k} \gamma_{k}=0, N^{-1} \sum_{k} \gamma_{k}^{2}=z^{-1}$.

\section{3. ФУНКЦИИ ГРИНА В ПРИБЛИЖЕНИИ ВЗАИМОДЕЙСТВУЮЩИХ МОД}

Приведенные в предыдущих разделах соотношения представляют собой систему тождеств, которые становятся уравнениями, если приближенно вычислить временны́ корреляционные функции (2.3), (2.4). Естественным приближением является приближение взаимодействующих мод, согласно которому спариваются разновременные операторы, входящие в (2.3) и (2.4). Однако при этом сразу же нарушаются основные правила сумм

$$
\frac{1}{2}-\sigma=\frac{1}{N} \sum_{k}\left\langle s_{k}^{-} s_{-k}^{+}\right\rangle, \quad \frac{1}{4}-\sigma^{2}=\frac{1}{N} \sum_{k}\left\langle\delta s_{k}^{z} \delta s_{-k}^{z}\right\rangle .
$$

В работах [9], [10] (см. также [8]) производилось умножение приближенного "массового оператора" на произвольный параметр, который использовался затем для того, чтобы обеспечить выполнение правил сумм. Такая процедура представляется достаточно грубой. В настоящей работе мы естественным образом введем в уравнения для функций Грина поперечной и продольной компонент спина параметры $a$ и $b$, воспользовавшись тем, что для $s_{q}^{+}$(и $s_{q}^{-}$) мы имеем два представления для свертки операторов $s_{k}^{z}$ и $s_{k}^{+}$(см. формулу (1.4)):

$$
\begin{aligned}
s_{k}^{+} & =\frac{2}{\sqrt{N}} \sum_{k_{1}} s_{k_{1}}^{z} s_{-k_{1}+k}^{+}=-\frac{2}{\sqrt{N}} \sum_{k} s_{-k_{1}+k}^{+} s_{k_{1}}^{z}= \\
& =\frac{1+a}{\sqrt{N}} \sum_{k_{1}} s_{k_{1}}^{z} s_{-k_{1}+k}^{+}-\frac{1-a}{\sqrt{N}} \sum_{k_{1}} s_{-k_{1}+k}^{+} s_{k_{1}}^{z},
\end{aligned}
$$

где параметр а считаем пока произвольным.

В (3.2) можно выделить намагниченность, положив

$$
\begin{aligned}
& s_{k}^{+}=2 \sigma s_{k}^{+}+\frac{2}{\sqrt{N}} \sum_{k_{1}} \delta s_{k_{1}}^{z} s_{-k_{1}+k}^{+}=\frac{2}{1-2 \sigma} \frac{1}{\sqrt{N}} \sum_{k_{1}} \delta s_{k_{1}}^{z} s_{-k_{1}+k}^{+}, \\
& s_{k}^{+}=-2 \sigma s_{k}^{+}-\frac{2}{\sqrt{N}} \sum_{k_{1}} s_{-k_{1}+k}^{+} \delta s_{k_{1}}^{z}=-\frac{2}{1+2 \sigma} \frac{1}{\sqrt{N}} \sum_{k_{1}} s_{-k_{1}+k}^{+} \delta s_{k_{1}}^{z} .
\end{aligned}
$$

В результате получим

$$
s_{k}^{+}=\frac{1+a}{1-2 \sigma} \frac{1}{\sqrt{N}} \sum_{k_{1}} \delta s_{k_{1}}^{z} s_{-k_{1}+k}^{+}-\frac{1-a}{1+2 \sigma} \frac{1}{\sqrt{N}} \sum_{k_{1}} \delta s_{k_{1}}^{z} s_{-k_{1}+k}^{+} .
$$

Представление (3.2) аналогично представлению Кэллена [11] для продольной компоненты спина,

$$
s_{k}^{z}=\frac{a}{2} \sqrt{N} \delta_{k 0}-\frac{1+a}{2 \sqrt{N}} \sum_{k_{1}} s_{k_{1}}^{-} s_{k_{1}+k}^{+}+\frac{1-a}{2 \sqrt{N}} \sum_{k_{1}} s_{k_{1}+k}^{+} s_{k_{1}}^{-},
$$


использовавшемуся для исследования спиновых систем вблизи нулевой температуры. Вводя вместо (3.5) представления (3.2), (3.4), мы пытаемся получить уравнения, пригодные в широком диапазоне температур, используя скрытую симметрию между компонентами спина, которая наиболее сильно проявляется в парамагнитной области и особенно в изотропном случае при $\xi=1$.

Используя (3.2) и (3.4), запишем уравнения движения (1.5), (1.7) в виде

$$
\begin{aligned}
i \dot{s}_{q}^{+}= & \left\{\omega_{q}^{T}+\frac{2 a+4 \sigma}{1-4 \sigma^{2}} \frac{I(0)}{N} \sum_{k}\left(\gamma_{k}-\xi \gamma_{-k+q}\right)\left\langle\delta s_{k}^{z} \delta s_{-k}^{z}\right\rangle\right\} s_{q}^{+}+\frac{I(0)}{N} \sum_{k, k_{1}}\left(\gamma_{k}-\xi \gamma_{-k+q}\right) \times \\
\times & \left\{\frac{1+a}{1-2 \sigma}: \delta s_{k}^{z} \delta s_{k_{1}}^{z} s_{-k_{1}-k+q}^{+}:-\frac{1+a}{1+2 a}: s_{-k_{1}-k+q}^{+} \delta s_{k}^{z} \delta s_{k_{1}}^{z}:\right\}, \\
i \dot{s}_{q}^{z}=i & \delta \dot{s}_{q}^{z}=-\frac{2 b+4 \sigma}{1-4 \sigma^{2}} \frac{\xi I(0)}{2 N} \sum_{k}\left(\gamma_{k}-\gamma_{-k+q}\right)\left\langle s_{k}^{-} s_{k}^{+}\right\rangle \delta s_{q}^{z}-\frac{\xi I(0)}{2 N} \sum_{k, k_{1}}\left(\gamma_{k}-\gamma_{-k+q}\right) \times \\
\times & \left\{\frac{1+b}{1-2 \sigma}: \delta s_{k_{1}}^{z} \delta s_{-k_{-}}^{-} s_{-k_{1}-k+q}^{+}:-\frac{1-b}{1+2 \sigma}: s_{-k_{-}}^{-} s_{-k_{1}-k+q}^{+} \delta s_{k_{1}}^{z}:\right\},
\end{aligned}
$$

где вновь точками выделены произведения операторов, спаривания которых запрещены. В (3.6) и (3.7) были использованы перестановочные соотношения (1.3) с целью сгруппировать операторы $s_{k}^{+}, s_{k}^{-}$отдельно от операторов $\delta s_{k}^{z}$ и $\delta s_{-k}^{z}$, кроме того, учтено следующее свойство функций $\gamma_{k}: \sum_{k} \gamma_{k}=0$. Подставляя теперь в $(2.3),(2.4)$ представления (3.6), (3.7), получим

$$
\begin{aligned}
\left(\left(i \dot{s}_{q}^{+} \mid\right.\right. & \left.\left.-i \dot{s}_{q}^{-}\right)\right)_{1, E}=\frac{I^{2}(0)}{N^{2}} \sum_{k, k_{1}, k^{\prime}, k_{1}^{\prime}}\left(\gamma_{k}-\xi \gamma_{-k+q}\right)\left(\gamma_{k^{\prime}}-\xi \gamma_{-k^{\prime}+q}\right) \times \\
\times & \left(\left(\frac{1+a}{1-2 \sigma}: \delta s_{k}^{z} \delta s_{k_{1}}^{z} s_{-k_{1}-k+q}^{+}:-\frac{1-a}{1+2 \sigma}: s_{-k_{1}-k+q}^{+} \delta s_{k}^{z} \delta s_{k_{1}}^{z}: \mid\right.\right. \\
& \left.\left.\frac{1+a}{1-2 \sigma}: s_{-k_{1}^{\prime}-k^{\prime}+q}^{-} \delta s_{-k_{1}^{\prime}}^{z} \delta s_{-k^{\prime}}^{z}:-\frac{1-a}{1+2 \sigma}: \delta s_{-k_{1}^{\prime}}^{z} \delta s_{-k^{\prime}}^{z} s_{-k_{1}^{\prime}-k^{\prime}+q}^{-}:\right)\right)_{1, E}, \\
\left(\left(i \dot{s}_{q}^{z} \mid-\right.\right. & \left.\left.i \dot{s}_{-q}^{z}\right)\right)_{1, E}=\frac{\xi^{2} I^{2}(0)}{4 N^{2}} \sum_{k, k_{1}, k^{\prime}, k_{1}^{\prime}}\left(\gamma_{k}-\gamma_{-k+q}\right)\left(\gamma_{k^{\prime}}-\gamma_{-k^{\prime}+q}\right) \times \\
\times\left(\left(\frac{1+b}{1-2 \sigma}: \delta s_{k_{1}}^{z} \delta s_{-k}^{-} s_{-k_{1}-k+q}^{+}:-\frac{1-b}{1+2 \sigma}: s_{-k^{-}}^{-} s_{-k_{1}-k+q}^{+} \delta s_{k_{1}}^{z}: \mid\right.\right. & \left.\left.\frac{1+b}{1-2 \sigma}: s_{-k_{1}^{\prime}-k^{\prime}+q^{-}}^{-} s_{-k_{1}^{\prime}}^{+} \delta s_{-k_{1}^{\prime}}^{z}:-\frac{1-b}{1+2 \sigma}: \delta s_{-k_{1}^{\prime}}^{z} s_{-k_{1}^{\prime}-k^{\prime}+q^{-k^{\prime}}}^{-} s_{-}^{-}\right)\right)_{1, E}
\end{aligned}
$$

Благодаря свойствам неприводимости (1.13) из выражений (3.8), (3.9) выпали входившие в (3.6), (3.7) члены, пропорциональные $s_{q}^{+}$и $\delta s_{q}^{z}$.

До сих пор все приведенные ранее соотношения были точными, а параметры $a$ и $b$ - произвольными. Производя в правых частях (3.8) и (3.9) спаривания разновременных операторов $\left\langle s_{k}^{+}(t) s_{k}^{-}\right\rangle,\left\langle s_{k}^{-}(t) s_{k}^{+}\right\rangle$и $\left\langle\delta s_{k}^{z}(t) s_{-k}^{z}\right\rangle$ и учитывая при этом, что величи́ны $\left\langle: \delta s_{k}^{z}(t) \delta s_{k_{1}}^{z}(t) s_{-k_{1}-k+q}^{+}(t): s_{q}^{-}\right\rangle, \ldots$ в выражениях для неприводимых 
функций (см. (1.13)) равны нулю, получаем “массовые операторы” (3.8), (3.9) в приближении взаимодействующих мод. С учетом спектральных представлений [3] выражения для них запишутся в виде

$$
\left(\left(i \dot{s}_{q}^{+} \mid-i \dot{s}_{q}^{-}\right)\right)_{1, E} \cong u^{2} M_{q}(E), \quad\left(\left(i \dot{s}_{q}^{z} \mid-i \dot{s}_{-q}^{z}\right)\right)_{1, E} \cong v^{2} \Pi_{q}(E)
$$

где

$$
\begin{aligned}
M_{q}(E)=\frac{I^{2}(0)}{2 N^{2}} \sum_{k, k_{1}}\left(\gamma_{k}+\gamma_{k_{1}}-\xi \lambda_{-k+q}-\xi \gamma_{-k_{1}+q}\right)^{2} \iint_{-\infty}^{\infty} d \omega d \omega^{\prime} d \omega^{\prime \prime} \times \\
\quad \times \frac{(1+n(\omega))\left(1+n\left(\omega^{\prime}\right)\right)\left(1+n\left(\omega^{\prime \prime}\right)\right)-n(\omega) n\left(\omega^{\prime}\right) n\left(\omega^{\prime \prime}\right)}{\left(\omega+\omega^{\prime}+\omega^{\prime \prime}\right)\left(E-\omega-\omega^{\prime}-\omega^{\prime \prime}\right)} \times \\
\quad \times \Psi_{k}(\omega) \Psi_{k_{1}}\left(\omega^{\prime}\right) \Phi_{-k_{1}-k+q}\left(\omega^{\prime \prime}\right), \\
\Pi_{q}(E)=\frac{\xi^{2} I^{2}(0)}{4 N^{2}} \sum_{k, k_{1}}\left(\gamma_{k}-\gamma_{-k+q}\right)^{2} \iint_{-\infty}^{\infty} d \omega d \omega^{\prime} d \omega^{\prime \prime} \times \\
\quad \times \frac{(1+n(\omega)) n\left(\omega^{\prime}\right)\left(1+n\left(\omega^{\prime \prime}\right)\right)-n(\omega)\left(1+n\left(\omega^{\prime}\right)\right) n\left(\omega^{\prime \prime}\right)}{\left(\omega-\omega^{\prime}+\omega^{\prime \prime}\right)\left(E-\omega+\omega^{\prime}-\omega^{\prime \prime}\right)} \times \\
\quad \times \Psi_{k_{1}}(\omega) \Phi_{k}\left(\omega^{\prime}\right) \Phi_{-k_{1}-k+q}\left(\omega^{\prime \prime}\right) .
\end{aligned}
$$

В формулах $(3.11),(3.12) \quad n(\omega)=\left(e^{\beta \omega}-1\right)^{-1}, \beta^{-1}=\theta=k T$, а спектральные плотности $\Phi_{k}(\omega)$ и $\Psi_{k}(\omega)$ равны

$$
\Phi_{k}(\omega)=-\frac{1}{\pi} \operatorname{Im}\left\langle\left\langle s_{q}^{+} \mid s_{q}^{-}\right\rangle\right\rangle_{\omega+i \varepsilon}, \quad \Psi_{k}(\omega)=-\frac{1}{\pi} \operatorname{Im}\left\langle\left\langle s_{k}^{z} \mid s_{-k}^{z}\right\rangle\right\rangle_{\omega+i \varepsilon},
$$

Функции Грина (2.5) и (2.6) в приближении взаимодействующих мод представляются в виде

$$
\begin{aligned}
\left\langle\left\langle s_{q}^{+} \mid s_{q}^{-}\right\rangle\right\rangle_{E} & =\frac{2 \sigma+u^{2} M_{q}(E)}{E-\left(2 \sigma+u^{2} M_{q}(E)\right) /\left(s_{q}^{+} \mid s_{q}^{-}\right)}, \\
\left\langle\left\langle s_{q}^{z} \mid s_{-q}^{z}\right\rangle\right\rangle_{E} & =\frac{v^{2} \Pi_{q}(E)}{E-v^{2} \Pi_{q}(E) /\left(s_{q}^{z} \mid s_{-q}^{z}\right)} .
\end{aligned}
$$

В правые части (3.10), (3.14) и (3.15) вошли параметры

$$
u=\frac{1+a}{1-2 \sigma}-\frac{1-a}{1+2 \sigma}=\frac{2 a+4 \sigma}{1-4 \sigma^{2}}, \quad v=\frac{1+b}{1-2 \sigma}-\frac{1-b}{1+2 \sigma}=\frac{2 b+4 \sigma}{1-4 \sigma^{2}}
$$

(см. (3.8), (3.9)). Их уже нельзя считать произвольными. В отличие от работ [9], [10] параметры $u$ и $v$ входят в уравнения естественным образом и имеют структуру, которая существенна при получении замкнутой системы уравнений для $a, b$ и $\sigma$.

На действительной оси, отделяя действительную и мнимую части, имеем $M_{q}(\omega)=$ $M_{q}^{\prime}(\omega)+i M_{q}^{\prime \prime}(\omega)$ и $\Pi_{q}(\omega)=\Pi_{q}^{\prime}(\omega)+i \Pi_{q}^{\prime \prime}(\omega)$. Используя уравнения (3.14) и (3.15), получаем приближенные выражения для спектральных плотностей:

$$
\Phi_{q}(\omega)=-\frac{1}{\pi} \frac{u^{2} M_{q}^{\prime \prime}(\omega) \omega}{\left\{\omega-\left(2 \sigma+u^{2} M_{q}^{\prime}(\omega)\right) /\left(s_{q}^{+} \mid s_{q}^{-}\right)\right\}^{2}+\left\{u^{2} M_{q}^{\prime \prime}(\omega) /\left(s_{q}^{+} \mid s_{q}^{-}\right)\right\}^{2}},
$$




$$
\Psi_{q}(\omega)=-\frac{1}{\pi} \frac{v^{2} \Pi_{q}^{\prime \prime}(\omega) \omega}{\left\{\omega-v^{2} \Pi_{q}^{\prime}(\omega) /\left(s_{q}^{z} \mid s_{-q}^{z}\right)\right\}^{2}+\left\{v^{2} \Pi_{q}^{\prime \prime}(\omega) /\left(s_{q}^{z} \mid s_{-q}^{z}\right)\right\}^{2}} .
$$

Выражения (3.14)-(3.18) в совокупности с выражениями (2.12) и (2.13) для восприимчивостей $\left(s_{q}^{+} \mid s_{q}^{-}\right)$и $\left(s_{q}^{z} \mid s_{-q}^{z}\right)$ представляют собой замкнутую систему уравнений. Используя спектральные представления корреляционных функций

$$
\left\langle s_{k}^{-} s_{k}^{+}\right\rangle=\int_{-\infty}^{\infty} d \omega\left(e^{\beta \omega}-1\right)^{-1} \Phi_{k}(\omega), \quad\left\langle\delta s_{-k}^{z} \delta s_{k}^{z}\right\rangle=\int_{-\infty}^{\infty} d \omega\left(e^{\beta \omega}-1\right)^{-1} \Psi_{k}(\omega),
$$

подставляя в них приближенные выражения (3.17), (3.18) и производя суммирование по $k$, получаем из соотношений (3.1) уравнения для определения параметров $u$ и $v$ (или $a$ и $b$ ). Усредняя гамильтониан (1.1) и учитывая (3.17)-(3.19), получаем приближенное выражение для средней энергии системы $\langle\mathcal{H}\rangle_{\theta}$, которую можно использовать для представления свободной энергии $F$ в виде

$$
F=E_{0}-\theta \int_{0}^{\theta} \frac{d \theta^{\prime}}{\theta^{\prime 2}}\left\langle\mathcal{H}-E_{0}\right\rangle_{\theta^{\prime}}
$$

где $E_{0}=-N\{I(0) / 8+\mu H / 2\}$. Уравнение для намагниченности $\sigma$ найдем из условия экстремума свободной энергии системы

$$
\frac{d F}{d \sigma}=0
$$

Вычисляя величины (3.19)-(3.21) с помощью приближенных спектральных плотностей (3.17), (3.18), мы должны учитывать структуру параметров $u$ и $v(3.16)$.

Уравнения, получающиеся из (3.1), и уравнение (3.21) представляют собой систему трансцендентных уравнений для определения параметров $\sigma, a$ и $b$, зависящих от температуры $\theta$. При $\sigma \rightarrow 0$ и $H=0$ они переходят в уравнения для критиче-

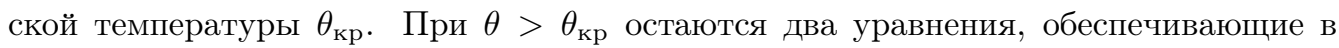
принятом приближении выполнение точных правил сумм $N^{-1} \sum_{k}\left\langle s_{k}^{-} s_{k}^{+}\right\rangle=1 / 2$ и $N^{-1} \sum_{k}\left\langle s_{k}^{z} s_{-k}^{z}\right\rangle=1 / 4$.

\section{Список литературы}

[1] Н. Н. Боголюбов, С. В. Тябликов, Докл. АН СССР, 126:1 (1959), 53-56.

[2] С. В. Тябликов, Методы квантовой теории магнетизма, Наука, М., 1965.

[3] Д. Н. Зубарев, УФН, 71:1 (1960), 71-116.

[4] Ю. А. Церковников, ТМФ, 49:2 (1981), 219-233.

[5] Ю. А. Церковников, ТМФ, 129:3 (2001), 432-463.

[6] R. Kubo, J. Phys. Soc. Japan, 12:6 (1957), 570-568.

[7] Ю. А. Церковников, ТМФ, 52:1 (1982), 147-160.

[8] Ю. А. Церковников, ТМФ, 40:2 (1979), 251-268.

[9] S. A. Scales, H. A. Gersch, J. Stat. Phys., 7:2 (1973), 95-118.

[10] K. Fujii, I. Mannari, S. Kadowaki, Progr. Theor. Phys., 50:5 (1973), 1501-1509.

[11] H. B. Callen, Phys. Rev., 130:3 (1963), 890-898. 